\title{
Aplicación de índices filogenéticos para la definición de prioridades de conservación en los páramos del noroeste de los Andes
}

\author{
Fernando Alzate $^{1 *}$, Astrid Álvarez ${ }^{1}$, Daniel Rafael Miranda-Esquivel ${ }^{2}$ \& Juan J. Morrone ${ }^{3}$ \\ 1. Grupo de Estudios Botánicos, Instituto de Biología, Universidad de Antioquia. Medellín, Colombia; \\ alveiro.alzate@udea.edu.co, ayasluna@gmail.com \\ 2. Laboratorio de Sistemática \& Biogeografía, Escuela de Biología, Universidad Industrial de Santander, Bucaramanga, \\ Colombia;dmiranda@uis.edu.co \\ 3. Museo de Zoología 'Alfonso L. Herrera', Departamento de Biología Evolutiva, Facultad de Ciencias, Universidad \\ Nacional Autónoma de México (UNAM), Ciudad de México, México; juanmorrone2001@yahoo.com.mx \\ * Correspondencia
}

Recibido 15-V-2018. Corregido 23-VII-2018. $\quad$ Aceptado 20-VIII-2018.

\begin{abstract}
Application of phylogenetic indices in the definition of conservation priorities in the northwest Andes paramos. Páramos are High Andean ecosystems that harbor a diverse biota and have high endemicity, because of their extreme environmental conditions. We used different phylogenetic indices to define conservation priorities in the paramos of the department of Antioquia, Colombia, based on measures of their evolutionary individuality and richness complimentary. To perform the analyses, we generated a database including 416 angiosperm taxa and 1951 localities for a total of 12897 distributional records compiled from surveys and reviews. Additionally, the available information in the Global Biodiversity Information Facility and the Colombian Biodiversity Information System were included. From these data, priority areas for the conservation of the paramos located in the state of Antioquia were identified, using measures of evolutionary individuality based on nodes and biotic complementarity. Taking into account only the individual phylogenetic indices, the most important paramo for conservation was Frontino-Urrao, followed by Farallones de Citará. If biotic complementarity is considered, the second most important paramo is Sonsón. Priority classifications of areas based on individuality are congruent with areas of greatest species richness. We conclude that it is necessary and urgent to preserve the paramos given their vulnerability and the subsequent loss of the ecosystem services that they provide, if they are exposed to degradation or disappearance.
\end{abstract}

Key words: Antioquia; Colombia; conservation; paramos; phylogenetic diversity; phylogenetic indices.

Alzate, F., Álvarez, A., Miranda-Esquivel, D. R. \& Morrone, J. J. (2018). Aplicación de índices filogenéticos para la definición de prioridades de conservación en los páramos del noroeste de los Andes. Revista de Biología Tropical, 66(4), 1353-1361.

La biodiversidad ha sido generalmente interpretada en términos ecológicos como el número y abundancia de las especies o táxones supraespecíficos en un sitio; ignorando los factores históricos subyacentes, y se debe considerar la biodiversidad como el producto de varios procesos tanto evolutivos como ecológicos. Así, cada vez que ocurre una extinción, las características evolutivas únicas de un linaje se pierden para siempre (Heard \& Mooers, 2000), por ello, la conservación de la biodiversidad requiere necesariamente tener en cuenta la historia evolutiva de los táxones (Posadas, Miranda-Esquivel, \& Crisci, 2001). Hipp et al. (2015) resumen las bondades de utilizar diferentes índices filogenéticos para la toma de decisiones de conservación de ecosistemas. Estos autores muestran cómo la comunidad 
científica debe adoptar la riqueza filogenética como un criterio indispensable para definir prioridades de conservación.

Los trabajos pioneros en proponer el uso de índices basados en información filogenética, plantearon que dicha información puede traducirse en un índice de diversidad taxonómica como una medida de su distinción (Vane-Wright, Humphries, \& Williams, 1991). Los índices propuestos por Vane-Wright et al. (1991), basados en los nodos de los cladogramas, solo toman en cuenta la posición ocupada por cada taxón en el cladograma, es decir, se fundamentan en la topología de los árboles filogenéticos, permitiendo asignar a los táxones un valor de conservación relativo a su historia evolutiva. Estos índices permiten asignar valores a las áreas que los táxones habitan, lo que posibilita establecer una jerarquía de prioridades de conservación donde el área más importante, y por lo tanto candidata para la conservación, es la que alberga la mayor biodiversidad, no significando esto el mayor número de especies, sino el mayor número de especies evolutivamente únicas (Posadas, Crisci, \& Katinas, 2011).

Existen varios índices que recogen distintas propiedades de la topología, el índice I es una medida de la información de la clasificación jerárquica que refleja el número de grupos a los cuales pertenece cada taxón (Vane-Wright et al., 1991) o el número de nodos compartidos (Freire, Morrone, \& Crisci, 1998). El índice I se refiere al número de grupos monofiléticos a los cuales pertenece un taxón y asigna a cada taxón un valor igual al de su grupo hermano; el cálculo se inicia asignando a los pares de terminales hermanos un valor arbitrario de 1. El índice $\mathrm{W}$ mide la información en una clasificación filogenética y fue diseñado específicamente para medir la proporción en que cada taxón contribuye a la diversidad total del grupo (Vane-Wright et al., 1991). El índice $\mathrm{W}$ se estima como la proporción en que cada taxón contribuye a la diversidad total del grupo, estableciendo un valor de información (i) para cada taxón terminal. El valor i se calcula como el número de nodos a los que pertenece cada taxón. Inicialmente se obtiene el peso $(\mathrm{Q})$ para cada taxón terminal, que es la proporción de diversidad total del grupo que es aportado por un taxón, donde $\mathrm{Q}$ para un taxón $\mathrm{j}$ es igual al sumatorio de los valores i dividido por el valor i de cada taxón $\mathrm{j}$ en el cladograma. El valor del índice $\mathrm{W}$ es obtenido para cada valor Q como la razón Qj estandarizada por el valor más bajo de Q para la totalidad del grupo (Posadas et al., 2001). Ambos índices dan prioridad a los terminales cercanos a la raíz en la filogenia, ya que ellos son filogenéticamente más informativos, por lo que las áreas ricas en ellos tendrán un valor mayor para la conservación. Estos índices dependen del sistema de estudio, por lo que no es posible comparar valores obtenidos de diferentes estudios, pero sí se puede comparar la ubicación relativa de las áreas de acuerdo con diferentes clasificaciones (Posadas et al., 2001).

La complementariedad (o el complemento del índice de Jaccard) entre áreas permite encontrar el conjunto mínimo de áreas que contenga el mayor porcentaje de la diversidad taxonómica total (Colwell \& Coddington, 1994). La complementariedad -al igual que el índice de Jaccard-, mide el número de especies no compartidas entre dos áreas; tiene un rango entre un valor máximo de 1 (no existen especies en común entre las áreas) y un valor mínimo de 0 (las áreas poseen las mismas especies); al ser usado como herramienta de priorización de áreas, posterior a la selección inicial de áreas dados los valores de los índices filogenéticos, permite minimizar el número de áreas protegidas, a la vez que se maximiza el número de especies conservadas (Posadas et al., 2001).

Los páramos son extensas regiones desarboladas que coronan las cúspides de las cordilleras por encima del bosque andino y generalmente se ubican por encima de los $3000 \mathrm{~m}$ (Cuatrecasas, 1958). Estos ecosistemas son fríos y húmedos, sufriendo cambios meteorológicos bruscos, casi siempre se hallan cubiertos por la niebla, poseen frecuentes precipitaciones y alta incidencia de vientos (Cuatrecasas, 1958). Cuatrecasas (1968) circunscribe los páramos a las "regiones elevadas 
y descubiertas de las cordilleras tropandinas en las que concurren especiales condiciones físicas, climáticas y meteóricas de tipo tropical, determinantes de formas particulares de vegetación". Las condiciones de clima y suelos determinan su presencia desde el norte del Perú, con algunas derivaciones llamadas jalcas (consideradas como regiones paramunas) hasta Ecuador, Colombia y Venezuela, y aisladamente en Panamá y Costa Rica (van der Hammen \& García, 2007). Los páramos en Colombia cuentan con una superficie total de 2905137 ha (Sarmiento, Cadena, Sarmiento, \& Zapata, 2013), que representa la mitad de la superficie total de este ecosistema a nivel mundial (Cleef, 2013) y el $2.5 \%$ del territorio emergido nacional (Corzo, 2013). Los páramos se caracterizan por presentar lluvias la mayor parte del año y una temperatura que puede fluctuar más de $10{ }^{\circ} \mathrm{C}$ entre el día y la noche, especialmente por encima de los $3800 \mathrm{~m}$ de altitud (Cleef, 2013). En términos generales se considera que el clima es principalmente húmedo, presentando precipitaciones entre $1000 \mathrm{y}$ $4000 \mathrm{~mm} / \mathrm{año}$, aunque se encuentran páramos secos cuyas precipitaciones están entre $600 \mathrm{y}$ $1000 \mathrm{~mm}$ anuales (Cleef, 2013).

El departamento de Antioquia, situado en el noroccidente de Colombia, posee seis complejos de páramo en las cordilleras de los Andes Centrales y Occidentales (Farallones de Citará, Frontino-Urrao, Paramillo, Belmira, Valle de Aburrá y Sonsón). El área que ocupan estos páramos es cercana a 46000 ha, las que representan menos del $1 \%$ del área de Antioquia (Alzate \& Murillo, 2016). Para los páramos de Colombia se han registrado 118 familias, 567 géneros y 3380 especies de espermatofitas (Rangel, 2000). De acuerdo con este autor, las familias más diversas son: Asteraceae, Orchidaceae, Poaceae, Melastomataceae y Bromeliaceae. Los géneros con mayor número de especies son: Epidendrum (105 especies), Espeletia (80), Pleurothallis (80) y Diplostephium (75). Para el caso de Antioquia, Alzate y Murillo (2016) estiman la diversidad de angiospermas de páramo en 693 especies, 277 géneros y 86 familias, siendo las familias más diversas: Asteraceae, Orchidaceae, Melastomataceae y Poaceae.

Actualmente los páramos están sometidos a un régimen de fuertes disturbios tanto naturales como antrópicos, con alta incidencia de alteraciones naturales, como grandes fenómenos geológicos (por ejemplo, actividad volcánica), erosión, lluvias, vientos, heladas, fuegos naturales y alteraciones producidas por animales (Van der Hammen \& Cleef, 1986). Por otro lado, las principales alteraciones antrópicas en el páramo son fuego, ganadería, agricultura (principalmente cultivos de papa), minería a cielo abierto y de socavón, plantaciones de especies exóticas, construcción de obras civiles, corte de matorrales para leña y especies invasoras (Sarmiento et al., 2013). Todas las formas de alteraciones antrópicas pueden causar la degradación o transformación de zonas de páramos, con lo que se perderían servicios ecosistémicos tan valiosos como la provisión y regulación de agua, pues un país como Colombia depende esencialmente de este tipo de ecosistemas (Hofstede, 2013). Este trabajo busca definir las prioridades de conservación para un conjunto de áreas de páramo ubicadas en la parte nororiental de los andes de Colombia.

\section{MATERIALES Y MÉTODOS}

Área de estudio: Se consideraron cinco áreas de páramo del departamento de Antioquia, Colombia, ubicadas en las cordilleras Central y Occidental de los Andes. Se excluyó del análisis el páramo de Paramillo, debido a la escasa información biológica que se tiene para el mismo. Los páramos de la cordillera Central incluidos fueron: Belmira, con altitudes entre 3100 y 3340 m (Alzate \& Murillo, 2016) y 10622 ha de extensión; Valle de Aburrá, con elevaciones máximas de $3250 \mathrm{~m}$ y una extensión de 870 ha; y Sonsón, con elevación máxima de $3340 \mathrm{~m}$ y 8707 ha de extensión (Fig. 1). De acuerdo con Alzate y Murillo (2016), estos paramos son florísticamente más afines entre sí que con los que se encuentran en la cordillera Occidental. De la cordillera Occidental se incluyeron Farallones de Citará, 


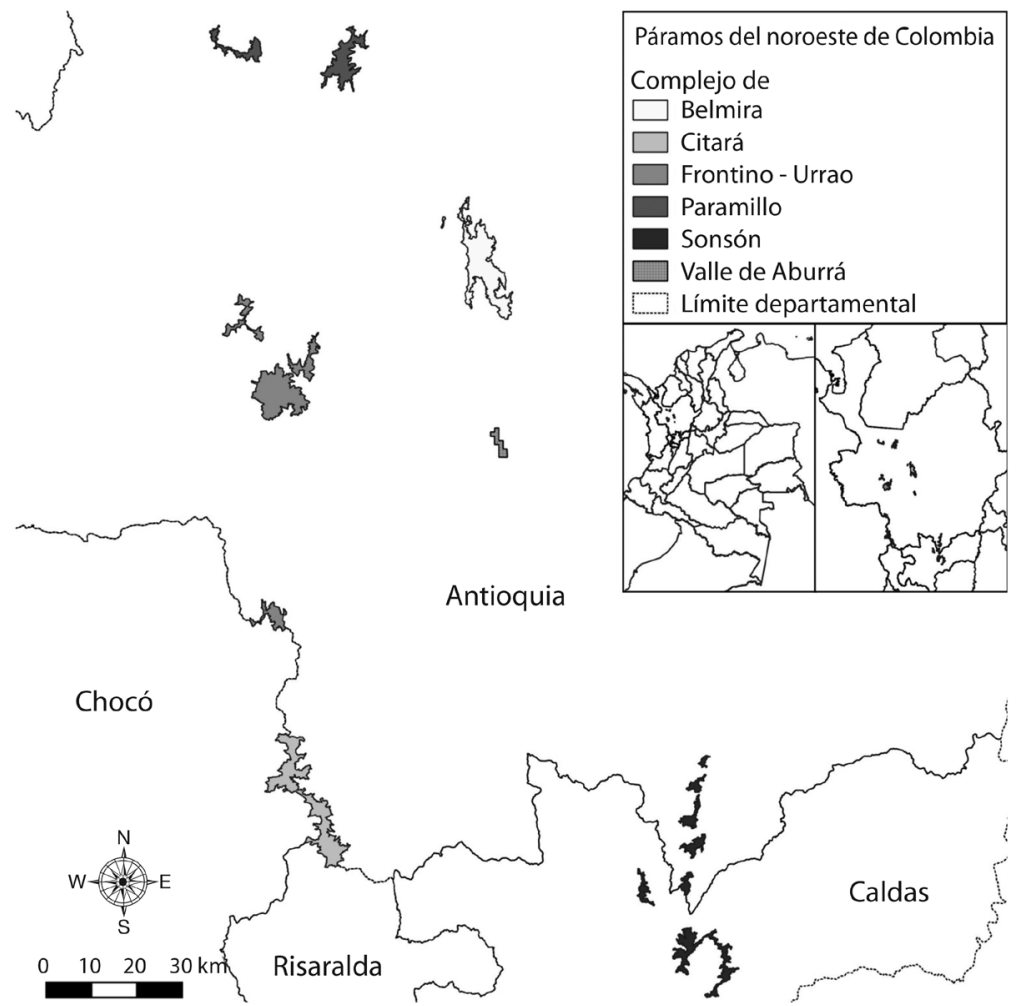

Fig. 1. Distribución de los cinco complejos de páramos existentes en el departamento de Antioquia, noroeste de Colombia (Elaboró Alex Espinosa).

CUADRO 1

Táxones empleados para calcular los índices filogenéticos I y W, con su respectiva referencia

\begin{tabular}{lll}
\multicolumn{1}{c}{ Familia } & \multicolumn{1}{c}{ Género } & \multicolumn{1}{c}{ Autor } \\
Alstroemeriaceae & Bomarea & Alzate et al., 2008 \\
Calceolariaceae & Calceolaria & Madriñan et al., 2013 \\
Campanulaceae & Lysipomia & Madriñan et al., 2013 \\
Celastraceae & Maytenus & McKenna et al., 2011 \\
Asteraceae & Diplostephium & Vargas y Madriñan, 2012 \\
Plantaginaceae & Plantago & Madriñan et al., 2013 \\
Rosaceae & Rubus & Lawrence y Campbell, 1999 \\
Rubiaceae & Arcytophyllum & Madriñan et al., 2013 \\
Valerianaceae & Valeriana & Bell y Donoghue, 2005 \\
\hline
\end{tabular}

con una extensión de 11233 ha y entre 3350 y $3970 \mathrm{~m}$ de altitud (Alzate \& Murillo, 2016); y Frontino-Urrao, con una extensión total de 13921 ha, entre 3400 y $3930 \mathrm{~m}$ de altitud (Morales et al., 2007).

Táxones: Se utilizaron las filogenias de nueve táxones de angiospermas, con un total de 416 especies (Cuadro 1). Los datos distribucionales se compilaron de proyectos anteriores (Alzate \& Murillo, 2016), de la Global Biodiversity Information Facility (GBIF.org 2016) y del Sistema de Información sobre Biodiversidad de Colombia (SIB, 2015). Se depuraron inconsistencias taxonómicas de los listados y de las distribuciones de los táxones 
en estas bases de datos. Se construyó una base de datos en la cual se discriminó taxón, continente, país y división administrativa por debajo de la categoría de país, así como la información de la localidad y sus coordenadas geográficas. Los datos encontrados sin coordenadas geográficas se ubicaron mediante GeoLocate (Ríos \& Bart, 2014). Todos los datos de distribución se transformaron a grados decimales y se mapearon para eliminar coordenadas marinas, empleando Arcgis.

El método de propincuidad media se aplicó a los datos de latitud-longitud, basado en el concepto de distancia con el vecino más próximo, con el objetivo de unir distribuciones cercanas. Finalmente, para realizar los análisis se generaron matrices especie-coordenadas para cada uno de los táxones.

Análisis de datos: El cálculo de la información contenida en las filogenias se realizó utilizando los nodos (Posadas et al., 2001). Los valores calculados I y W para cada especie que habita un área en particular se usaron para calcular el valor de cada área (Posadas et al., 2001). Los índices se estandarizaron con la finalidad de controlar la sobreponderación de clados que tienen un gran número de táxones (índices Is y Ws) (Posadas et al., 2001); cuando un taxón estuvo presente en dos o más áreas, se asignó el valor del índice a las áreas donde estaba presente (índices I y W) o se estandarizo de acuerdo al número de áreas donde estaba presente (Ie y We), o se estandarizo usando tanto la distribución de las especies como el número de terminales de cada topología (Ise y Wse) (Posadas et al., 2001).

Adicionalmente, se calculó la riqueza para cada área y la complementariedad entre áreas, medido como el complemento del índice de similitud de Jaccard (Colwell \& Coddington, 1994). Los cálculos se realizaron utilizando los programas Jrich (Miranda-Esquivel 2016; R Core Team, 2016) y EstimateS (Colwell, 2013).

\section{RESULTADOS}

El análisis inicial se realizó con un total de 416 táxones. Los táxones que sustentan el valor total de los índices para el páramo Frontino-Urrao representan el $5.8 \%$ del total de táxones, para Farallones de Citará el $3.1 \%$, para Valle de Aburrá el $1.9 \%$, y para Belmira y Sonsón el $1.7 \%$.

En la clasificación de los páramos según el índice I, Frontino-Urrao ocupa la primera posición, seguido de Belmira, Farallones de Citará, Valle de Aburrá y Sonsón en la última posición. Al considerar el índice W, FrontinoUrrao obtiene el mayor valor, seguido de Farallones de Citará, Belmira, Valle de Aburrá y como ocurrió con el índice I, Sonsón obtuvo también el más bajo valor. Con los índices estandarizados Is y Ws, la clasificación de los páramos presentó a Frontino-Urrao con el valor más alto, seguido de Belmira, Farallones de Citará, Sonsón, y los valores más bajos fueron para Valle de Aburrá.

Índice I: Teniendo en cuenta solo los páramos de Antioquia, el peso total del índice I clasifica las zonas de páramo de mayor a menor prioridad de conservación así: Frontino-Urrao, Belmira, Farallones de Citará, Valle de Aburrá y Sonsón (Cuadro 2). Incluyendo endemicidad (Ie), la clasificación cambia notoriamente, solo se mantiene Frontino-Urrao en la primera posición, seguido de Farallones de Citará, lo cual es debido posiblemente a que fue la única área donde se encontró una especie endémica, que aportó un valor más alto al índice (Ie). Los índices I e Ie tuvieron una alta correlación $(\mathrm{r}=$ $0.86, \mathrm{P}<0.05$ ).

Las tres primeras posiciones mantuvieron el mismo orden tanto en la clasificación con el índice I neto como en el estandarizado (Cuadro 2), solo cambió en la segunda posición cuando se tiene en cuenta la estandarización del índice Ie. Los índices estandarizados Is e Ise presentaron un orden de priorización similar para la conservación de los páramos de Antioquia, ubicando en el primer lugar a Frontino-Urrao, seguido de Belmira, Farallones de Citará, Sonsón y Valle de Aburrá (Cuadro 2).

Índice W: Con base en este índice, Frontino-Urrao es el área de mayor prioridad de 
CUADRO 2

Índice I y W con sus diferentes estimaciones, endemicidad, riqueza y área de cada páramo

\begin{tabular}{lccccccccccc}
\multicolumn{1}{c}{ Páramo } & I & Ie & Is & Ise & W & We & Ws & Wse & Riqueza & Endemicidad & Área (ha) \\
Frontino-Urrao & 67 & 3.137 & 0.379 & 0.022 & 35.75 & 1.894 & 0.199 & 0.012 & 24 & 0 & 13921 \\
Farallones de Citará & 33 & 2.389 & 0.141 & 0.01 & 18.83 & 2.647 & 0.064 & 0.011 & 13 & 1 & 11233 \\
Belmira & 34 & 1.844 & 0.220 & 0.017 & 13.03 & 0.743 & 0.073 & 0.006 & 7 & 0 & 10622 \\
Valle de Aburrá & 27 & 0.858 & 0.118 & 0.004 & 10.65 & 0.437 & 0.032 & 0.001 & 8 & 0 & 1500 \\
Sonsón & 23 & 0.922 & 0.124 & 0.005 & 9.552 & 0.554 & 0.06 & 0.004 & 7 & 0 & 8707 \\
\hline
\end{tabular}

Índices crudos: (I, W); índices estandarizados: (Is, Ws); índices divididos por el número de áreas donde el taxón ocurre: (Ie, We); ponderación del estandarizado por áreas de presencia: (Ise, Wse).

conservación, seguido de Farallones de Citará, Belmira, Valle de Aburrá y Sonsón. Considerando la endemicidad en este índice, el páramo más prioritario a conservar fue Farallones de Citará seguido de Frontino-Urrao (Cuadro 2). Al comparar la clasificación arrojada entre el índice $\mathrm{W}$ crudo y estandarizado, se observa que las prioridades de conservación se modificaron para las últimas cuatro áreas de páramo, invirtiéndose el orden de priorización entre los dos índices (Cuadro 2). Farallones de Citará fue el área más prioritaria a conservar considerando la endemicidad, pero al estandarizar este índice, ocupó el segundo lugar después de Frontino-Urrao. El orden de priorización con los índices crudos (I y W) solo difiere en la segunda posición, ya que con el índice I se ubicó al páramo de Belmira y con el índice W, a Farallones de Citará (Cuadro 2). Los pares de áreas con valores más altos de complementariedad son Valle de Aburrá - Farallones de Citará con 0.895 y Sonsón - Farallones de Citará con 0.889 (Cuadro 3).

Con respecto a la riqueza, el páramo de Frontino-Urrao obtuvo el mayor valor, con 24 especies (Cuadro 2), posición que también ocupó con los demás índices estimados.
El índice $\mathrm{W}$ y la riqueza presentan una alta correlación $(\mathrm{r}=0.94 ; \mathrm{P}<0.05)$, lo que indica que son indicadores con información similar para clasificar las áreas. El tamaño del área y los índices estimados para cada páramo no tuvieron alta correlación. La endemicidad en Farallones de Citará fue de $7.7 \%$ y cero para los restantes páramos, ya que todas las especies incluidas en el análisis son de distribución amplia. En el repositorio: [https://github.com/ Dmirandae/paramo] se encuentran los datos iniciales y un ejemplo de las corridas.

\section{DISCUSIÓN}

En este estudio no se encontraron diferencias marcadas entre los resultados obtenidos con los diferentes índices aplicados. Algunos autores plantean que "la jungla de índices filogenéticos" puede ser utilizada tanto para definir prioridades de conservación como para explicar los patrones de la diversidad filogenética (Winter, Devictor, \& Schweiger, 2013). Los índices aquí aplicados permiten calificar y definir las prioridades de cada una de las áreas, mediante diferentes fuentes de datos. La inclusión de los índices I y W permite tener el

CUADRO 3

Complementariedad de especies entre los cinco páramos evaluados

\begin{tabular}{lcccc} 
& Farallones de Citará & Sonsón & Belmira & Valle de Aburrá \\
Sonsón & 0.889 & & & \\
Belmira & 0.824 & 0.833 & 0.846 & 0.851 \\
Valle de Aburrá & 0.895 & 0.636 & 0.76 & 0.76 \\
Frontino-Urrao & 0.63 & 0.853 & \\
\hline
\end{tabular}


componente de diversidad filogenética de cada linaje en la evaluación de prioridades, mientras que la diversidad alfa estaría considerada por la riqueza, la endemicidad constituye un indicativo de la diversidad beta. Se puede afirmar que los análisis realizados permiten evaluar e incluir la mayoría de las aristas implicadas en la conservación biológica.

Todos los índices, excepto We, presentaron a Frontino-Urrao como el páramo con la mayor prioridad de conservación, siendo el área que posee la mayor cantidad de especies y extensión y un bajo grado de transformación antrópica (Rivera \& Pinilla, 2014). Si bien el páramo de Frontino-Urrao es uno de los páramos con menor porcentaje de transformación, se debe resaltar que no se encuentra bajo ninguna categoría de protección especial, lo que lo hace vulnerable al cambio de su cobertura vegetal, perdiéndose información filogenética única para siempre.

Los índices I y W tuvieron algunas variaciones en la clasificación de los páramos, pero se debe considerar que $\mathrm{W}$ evita el problema de sobreestimación, que se presenta con el índice I (Posadas et al., 2001). Lysipomia muscoides Hook. f. fue la especie que aportó más peso al índice I para Belmira, pero con el índice $\mathrm{W}$ el peso de dicha especie disminuyó considerablemente. Al disminuir este valor, el aporte de los táxones presentes en Farallones de Citará que tenían bajo valor con el índice I, fue más significativo y este páramo obtuvo el mayor valor con el índice W, con lo cual se muestra que el índice $\mathrm{W}$ reduce la sobrestimación del índice I. En algunos casos los índices no fueron coherentes en la priorización de las áreas. Esto se advierte en las posiciones que tuvieron Sonsón y Valle de Aburrá, lo cual no permite tener una decisión clara respecto a su clasificación. La estandarización evitó que táxones pertenecientes a filogenias con pocas terminales sufrieran subvaloración con respecto a los que pertenecen a táxones más diversos. La estandarización de los índices se realizó con el fin de comparar topologías de diferente tamaño, disminuyendo el peso de los árboles con muchos terminales (Posadas et al., 2001). Sin embargo, de acuerdo con Roig-Juñent y Debandi (2004), la estandarización genera que los árboles con pocas especies tengan un alto valor, y esto podría estar sucediendo en este estudio, con la estandarización del índice $\mathrm{W}$, e incluso del índice I, donde el área de mayor prioridad fue Belmira y no Farallones de Citará, debido probablemente a que dentro de las especies que soportan el valor total Ws en Belmira se encuentra Lysipomia muscoides, especie incluida en un cladograma con pocos terminales.

Los índices filogenéticos no presentan alta correlación con la extensión de los páramos, aunque en este análisis de forma casual, el área de mayor extensión (Frontino-Urrao), fue también la de mayor prioridad para la conservación según los índices I y W. Los resultados del índice de complementariedad presentan a Sonsón como el área más complementaria al páramo Frontino-Urrao, la cual es la de mayor prioridad de conservación, como se puede advertir en el cuadro 3 .

El incorporar la diversidad filogenética en la restauración y conservación de áreas, permite evitar la pérdida de la estructura filogenética y con ello mantener el adecuado equilibrio de los ecosistemas (Begley-Miller, Hipp, Brown, Hahn, \& Rooney, 2014). Este trabajo abordó diferentes metodologías para plantear prioridades de conservación de áreas, mediante métodos basados en información filogenética que consideran el componente evolutivo de la diversidad y permiten la identificación de aquellas áreas que garanticen la preservación de las especies potencial y filogenéticamente raras.

\section{AGRADECIMIENTOS}

Expresamos nuestro agradecimiento a Sebastián González por su considerable apoyo en los análisis estadísticos y filogenéticos. Así mismo al personal del Herbario de la Universidad de Antioquia (HUA), especialmente a Felipe Cardona y Marcela Calderón por su ayuda en temas taxonómicos y cartográficos. Agradecemos también el considerable trabajo y aporte de Angélica Arcila para mejorar este manuscrito. Este proyecto fue realizado con 

el financiamiento del CODI de la Universidad
de Antioquia.

\section{RESUMEN}

Los páramos son ecosistemas andinos de alta montaña, que albergan una biota diversa y de alta endemicidad, producto de las condiciones ambientales extremas. Se utilizaron diferentes índices para definir prioridades de conservación en las áreas de páramo del departamento de Antioquia, Colombia; con base en medidas de la individualidad evolutiva y complementariedad de biotas. Para realizar los análisis se generó una base de datos que incluyó 416 táxones de angiospermas y 1951 localidades, para un total de 12897 registros de distribución, compilados a partir de los muestreos y revisiones realizados en este proyecto; adicionalmente se incluyó la información disponible en Global Biodiversity Information Facility y en el Sistema de Información sobre Biodiversidad de Colombia. A partir de estos datos se identificaron áreas de prioridad para la conservación de los páramos de Antioquia, de acuerdo con medidas de la individualidad evolutiva basadas en nodos y complementariedad de biotas. Teniendo en cuenta solamente los índices de individualidad evolutiva, el páramo prioritario para la conservación fue Frontino-Urrao, seguido de Farallones de Citará; pero si se considera la complementariedad de biotas, la segunda área prioritaria para la conservación es Sonsón. Las clasificaciones de prioridad de áreas basadas en individualidad son congruentes con las áreas de mayor riqueza de especies. Es necesario y urgente preservar el ecosistema páramo dado su vulnerabilidad y la subsecuente perdida de los servicios ecosistémicos que brindan, si se ven expuestos a la degradación o desaparición.

Palabras claves: Antioquia; Colombia; conservación; diversidad filogenética; índices filogenéticos; páramos.

\section{REFERENCIAS}

Alzate, F., Mort, M., \& Ramírez, M. (2008). Phylogenetic analyses of Bomarea (Alstroemeriaceae) based on combined analyses of nrDNA ITS, psbA - trnH, rpoB - trnC y matK sequences. Taxon, 57, 1-10.

Alzate, F., \& Murillo, J. (2016). Angiosperm flora on the páramos of northwestern Colombia: Diversity and affinities. PhytoKeys, 70, 41-52.

Begley-Miller, D., Hipp, A., Brown, B., Hahn, M., \& Rooney, T. (2014). White-tailed deer are a biotic filter during community assembly, reducing species and phylogenetic diversity. AoB Plants, 6, plu030.

Bell, C., \& Donoghue, M. (2005). Phylogeny and biogeography of Valerianaceae (Dipsacales) with special reference to the South American valerians. Organisms Diversity \& Evolution, 5, 147-159.

Cleef, A. (2013). Origen, evolución, estructura y diversidad biológica de la alta montaña colombiana. En J. Cortés-Duque \& C. Sarmiento (Eds.), Visión socioecosistémica de los páramos y la alta montaña colombiana: Memorias del proceso de definición de criterios para la delimitación de páramos (pp. 3-21). Bogotá: Instituto de Investigación de Recursos Biológicos Alexander von Humboldt.

Colwell, R. (2013). Estimates: Statistical estimation of species richness and shared species from samples. Retrieved from http://viceroy.eeb.uconn.edu/ estimates/

Colwell, R., \& Coddington, J. A. (1994). Estimating terrestrial biodiversity through extrapolation. Philosophical Transactions of the Royal Society of London Series B, 345, 101-118.

Corzo, G. (2013). Una mirada desde los páramos a la conservación de la biodiversidad en Colombia. En J. Cortés-Duque \& C. Sarmiento (Eds.), Visión socioecosistémica de los páramos y la alta montaña colombiana: Memorias del proceso de definición de criterios para la delimitación de páramos (pp. 89-102). Bogotá: Instituto de Investigación de Recursos Biológicos Alexander von Humboldt.

Cuatrecasas, J. (1958). Aspectos de la vegetación natural de Colombia. Revista de la Academia Colombiana de Ciencias Exactas, Físicas y Naturales, 10, 221-264.

Cuatrecasas, J. (1968). Páramo vegetation and its life forms: Geoecology of the mountainous regions of the tropical Americas. Colloquium Geographicum, 9, 163-183.

Freire, S., Morrone, J. J., \& Crisci, J. V. (1998). Cladistic measures for conservation in southern South America: A view from the Asteraceae (angiosperms) and Curculionidae (Insecta: Coleoptera). Physis, 55, 27-32.

GBIF.org. (2016). GBIF Home Page. Retrieved from http:// gbif.org

Heard, S., \& Mooers, A. (2000). Phylogenetically patterned speciation rates and extinction risks change the loss of evolutionary history during extinctions. Proceedings of the Royal Society of London, 267, 613-620.

Hipp, A. L., Larkin, D. J., Barack, R. S., Bowles, M. L., Cadotte, M. W., Jacobi, S. K., ... Weiher, E. (2015). On the nature of things: Phylogeny in the service of ecological restoration. American Journal of Botany, $102,497-498$.

Hofstede, R. (2013). Lo mucho que sabemos del páramo. Apuntes sobre el conocimiento actual de la integridad, la transformación y la conservación del páramo. En J. Cortés-Duque \& C. Sarmiento (Eds.), Visión 
socioecosistémica de los páramos y la alta montaña colombiana: memorias del proceso de definición de criterios para la delimitación de páramos (pp. 113125). Bogotá: Instituto de Investigación de Recursos Biológicos Alexander von Humboldt.

Lawrence, A., \& Campbell, C. (1999). Phylogeny of Rubus (Rosaceae) based on nuclear ribosomal DNA internal transcribed spacer region sequences. American Journal of Botany, 86, 81-97.

Madriñan, S., Cortes, A., \& Richardson, J. (2013). Páramo is the worlds's fastest evolving and coolest biodiversity hotspot. Frontiers in Genetics, 4,192.

McKenna, M., Simmons, M., Bacon, C., \& Lombardi, J. (2011). Delimitation of segregate genera of Maytenus s.l. (Celastraceae) based on morphological and molecular characters. Systematic Botany, 36, 922-932.

Miranda-Esquivel, D. R. (2016). Support in area prioritization using phylogenetic information. In R. Pellens, \& P. Grandcolas (Eds.), Biodiversity conservation and phylogenetic dystematics: Preserving our evolutionary heritage in an extinction crisis (pp. 219-235). Springer. DOI: 10.1007/978-3-319-22461-9

Morales, M., Otero, J., Van der Hammen, T., Torres, A., Cadena, C., Pedraza, C., ... Cardenas, L. (2007). Atlas de los Páramos de Colombia. Bogotá: Instituto de Investigación de Recursos Biológicos Alexander von Humboldt.

Posadas, P., Crisci, J. V., \& Katinas, L. (2011). Sistemática y biogeografía en la conservación de la biodiversidad: ejemplos de América del Sur austral. En J. Simonetti, \& R. Dirzo (Eds.), Conservación biológica: Perspectivas desde América Latina (pp. 109-125). Santiago de Chile: Editorial Universitaria.

Posadas, P., Miranda-Esquivel, D. R., \& Crisci, J. V. (2001). Using phylogenetic diversity measures to set priorities in conservation: An example from southern South America. Conservation Biology, 15, 1325-1334.

R Core Team (2016). $R$ : A language and environment for statistical computing. R Foundation for Statistical Computing. Viena, Austria. Recuperado de http:// www.R-project.org/

Rangel, C. (2000). La región de vida paramuna. Colombia Diversidad Biótica III. Bogotá: Universidad Nacional de Colombia, Instituto de Ciencias Naturales e Instituto Alexander von Humboldt.
Rios, N. E., \& Bart, H. L. (2014). GEOLocate (Version 3.22). Belle Chasse, LA: Tulane University Museum of Natural History.

Rivera, D., \& Pinilla, C. (2014). Transformación de los páramos en Colombia. En M. Cabrera \& W. Ramírez (Eds.), Restauración ecológica de los páramos de Colombia: Transformación y herramientas para su conservación (pp. 63-65). Bogotá: Instituto de Investigación de Recursos Biológicos Alexander von Humboldt.

Roig-Juñent, S., \& Debandi, G. (2004). Prioridades de conservación aplicando información filogenética y endemicidad: Un ejemplo basado en Carabidae (Coleoptera) de América del Sur austral. Revista Chilena de Historia Natural, 77, 695-709.

Sarmiento, C., Cadena C., Sarmiento M., \& Zapata, J. (2013). Aportes a la conservación estratégica de los páramos de Colombia: Actualización de la cartografía de los complejos de páramo a escala 1:100.000. Bogotá: Instituto de Investigación de Recursos Biológicos Alexander von Humboldt.

SiB Colombia. (2016). Sistema de Información sobre Biodiversidad de Colombia. Recuperado de www. sibcolombia.net/web/sib/home

Van der Hammen, T., \& Cleef, A. (1986). Development of the high Andean paramo flora and vegetation. In F. Vuilleumier \& M. Monasterio (Eds.), High altitude tropical biogeography (pp. 153-201). Oxford: University Press.

Van der Hammen, T., \& García, J. (2007). Los páramos: Archipiélagos terrestres en el norte de los Andes. En M. Morales, J. Otero, T. Van der Hammen, A. Torres, C. Cadena, N. Pedraza-Rodríguez, ... L. Cárdenas (Eds.), Atlas de páramos de Colombia (pp. 25-31). Bogotá: Instituto de Investigación de Recursos Biológicos Alexander von Humboldt.

Vane-Wright, R. I., Humphries, C. J., \& Williams, P. H. (1991). What to protect? Systematics and the agony of choice. Biological Conservation, 55, 235-254.

Vargas, O., \& Madriñan, S. (2012). Preliminary phylogeny of Diplostephium (Asteraceae): Speciation rate and character evolution. Lundellia, 15, 1-15.

Winter, M., Devictor, V., \& Schweiger, O. (2013). Phylogenetic diversity and nature conservation: Where are we? Trends in Ecology \& Evolution, 28, 199-204. 